\title{
Analysis of the sensory threshold between paretic and nonparetic sides for healthy rehabilitation in hemiplegic patients after stroke
}

\author{
Hye-Joo Jeon ${ }^{1}$, Ju-Hyun Kim¹, Byong-Yong Hwang ${ }^{2}$, Bokyung Kim³ ${ }^{3}$ Junghwan Kim ${ }^{2}$ \\ ${ }^{1}$ Laboratory of Health Science \& Nanophysiotherapy, Department of Physical Therapy, Graduate Scool, Yongin University, Yongin, \\ Korea \\ ${ }^{2}$ Department of Physical Therapy, College of Public Health \& Welfare, Yongin University, Yongin, Korea; \\ *Corresponding Author: junghwankim3@yongin.ac.kr \\ ${ }^{3}$ Department of Physiology, Institute of Functional Genomics, School of Medicine, Konkuk University, Seoul, Korea
}

Received 27 October 2012; revised 30 November 2012; accepted 5 December 2012

\begin{abstract}
The purpose of this study was to investigate the differences in the sensory threshold between the paretic and nonparetic sides of hemiplegic patients. 28 patients who were hemiplegic poststroke (14 men and 14 women) participated in the electrical sensory and pain thresholds study; 22 patients who were hemiplegic post-stroke (13 men, 9 women) participated in a study measureing the sensory threshold with light touch. Electrical sensory and pain thresholds were measured in the forearm via transcutaneous electrical nerve stimulation. The light-touch threshold was measured in the forearm using monofilaments. The light-touch, electrical sensory, and pain thresholds for the paretic side were significantly higher than for the nonparetic side in our population, respectively. In both the nonparetic and paretic sides, the male group generally showed higher thresholds for pain and sensation than did the female group. These results suggest that the different evaluations of sensory thresholds performed in this study for healthy rehabilitation will be a valuable clinical tool in hemiplegic patients after stroke.
\end{abstract}

Keywords: Sensory Threshold; Paretic and Nonparetic Sides; Healthy Rehabilitation; Stroke

\section{INTRODUCTION}

Sensory impairment is common following stroke [1]. After stroke, a loss of physical connections (synapses, dendrites, axons) linking brain regions appears from impairment to the axons to the infarct site [2]. A correlation between after stroke motor function and structural integ- rity of the corticospinal tract has been found [3,4]. The sensory element of the superior thalamic radiation (sSTR) contains all afferent links to the somatosensory cortex [5]. Borstad et al. suggested that stroke associated structural changes to the sSTR may have relation to after stroke sensory function [2]. It is important to build an understanding of discriminative sensory impairment because this type of subtle sensory disorder might be related to functional outcomes in patients who are rehabilitating after a stroke [6,7]. In many studies, it has been established that sensory impairment is detrimental to motor recovery $[8,9]$. Poor functional recovery may be partially result from learned nonuse phenomenon and bring about degeneration of motor function [10,11]. Tyson et al. found that sensory impairment was significantly associated with mobility or lack thereof, independence in recovery, and activities of daily living [12]. Also, sensory impairment indicates to predict length of hospital stay and discharge placement [2]. Measuring the prevalence and severity of sensory loss, particularly in patients who present for treatment and accurate detection of this loss, is essential [13]. Better understanding of impairments and outcomes can improve the established clinical pathways and facilitate better timing and allocation of rehabilitation [14]. However, conventional sensory testing is insufficient for exact assessment of the amount of sensory impairment in patients [15]. The word threshold refers to the points of stimulus strength at which the participant first notices the stimulation at all and as painful, respectively $[16,17]$. Sensory acuity is most generally determined by a threshold test. The functional part of sensory acuity is often forecast by using discrimination tests that evaluate the quality of sensation [18]. In addition, the assessment of change in a given sensory threshold could be a profitable tool in the clinical evaluation of pain [19]. The electrical sensory threshold is significant 
because the therapist can regulate treatment intensity from their patient's subjective sensation during use of a transcutaneous nerve electrical stimulator [20]. Recently, experimental and clinical investigations have demonstrated some parameters for the electrical sensory threshold in healthy volunteers $[19,21,22]$. However, there are few clinical studies demonstrating the sensory threshold in patients after a stroke. Unilateral cerebral hemisphere lesions can cause sensory impairment on the nonparetic side [23,24]. Corkin et al. asserted that lesion size was a demonstrable factor in sensory impairment on the nonparetic side [25]. Hemiplegic patients with ipsilateral as well as contralateral tactile impairment face considerable additional rehabilitation challenges [26]. Therefore, more study is needed to evaluate the nonparetic side of a hemiplegic patient, and in particular to focus on the comparison between the paretic and nonparetic sides. To our knowledge, no previous study of sensory and pain thresholds exists that specifically applies to the differences between the paretic and nonparetic sides of a hemiplegic patient. This study aims to evaluate three different sensory thresholds (light-touch, electrical sensory, and pain) in measuring and comparing these differences.

\section{MATERIALS AND METHODS}

\subsection{Subjects}

The patients were selected according to the following inclusion criteria: left or right hemisphere stroke (unilateral only); a roughly equivalent number of patients from both sexes; ability to communicate and understand instructions (Table 1).

\subsection{Electrical Sensory and Pain Thresholds}

28 patients who were hemiplegic post-stroke (14 men and 14 women) participated in the study. Patients were comfortably seated on a physical therapy treatment table or a wheelchair with their upper extremities positioned on a pillow. In each patient, the forearm was stimulated using the transcutaneous nerve electrical stimulator (HAT2000, Meditens Co. Ltd., Korea) and two surface electrodes of the same size $(4.5 \times 6 \mathrm{~cm})$ for bipolar stimulation. The forearm was placed in a pronated position and effectively divided into thirds for the purpose of determining electrode position, with one electrode placed on the proximal aspect of the forearm at one-third of the forearm length, and the other placed at the distal aspect at one-third of the length (Figure 1(a)). The electrical stimulation doesn't use the heat of the electrode. The pad does get enough water during every treatment procedure. The electrical stimulation was used for $10 \mathrm{~Hz}$ frequency in "mode 1-tapping". The current intensity was recorded as $0 \%$ to $100 \%$ of the total output, and the sensory threshold was gradually increased by increasing the intensity. The first threshold was measured when the patients perceived the electrical stimulation without pain. The stimulus was then continuously increased in intensity until the patient felt initial pain. The stimulation was considered to be over the sensory threshold for pain when the patient felt sick or sore. This method was first performed in the paretic and then in the nonparetic side for each patient.

\subsection{Light-Touch Threshold}

22 patients who were hemiplegic post-stroke (13 men and 9 women) participated in the study. Touch thresholds

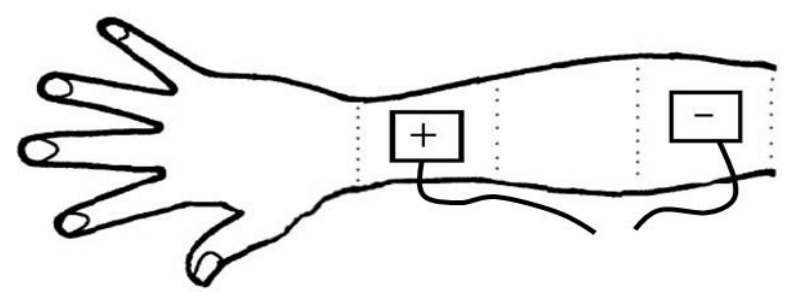

(a) Sensory \& pain threshold

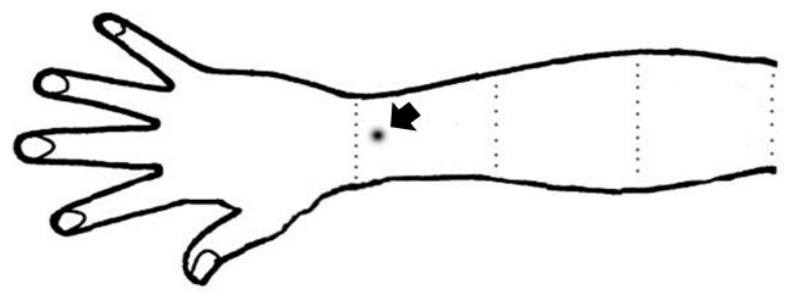

(b) Light touch threshold

Figure 1. Schematic representation of the experimental methods for measuring threshold.

Table 1. Clinical characteristics of patients with after stroke.

\begin{tabular}{cccccc}
\hline Gender (\%) & Age (yr) & Height $(\mathrm{cm})$ & Weight $(\mathrm{kg})$ & BMI $\left(\mathrm{kg} / \mathrm{m}^{2}\right)$ & Hypertension $(\%)$ \\
\hline Male 22 (54) & $50.2 \pm 14.9$ & $164.2 \pm 7.8$ & $62.3 \pm 10.5$ & $23.1 \pm 3.0$ & Paretic side \\
& DM (\%) & Time after stroke (mo) & Right (\%) & Left (\%) \\
Female 19 (49) & $4(9)$ & $3.6 \pm 2.0$ & $15(37)$ & $26(63)$ \\
\hline
\end{tabular}

BMI, body mass index; DM, diabetes mellitus. 
were measured using monofilaments. The test kit consisted of 5 different filaments; bending pressure ranges for the individual filaments were 2.83 to $0.07 \mathrm{~g}, 3.61$ to $0.4 \mathrm{~g}, 4.31$ to $2.0 \mathrm{~g}, 4.56$ to $4.0 \mathrm{~g}$, and 6.65 to $300 \mathrm{~g}$. To perform the test, the monofilament must be applied (that is, be pressed until it bends) perpendicular to the skin and held in place for 1 to 1.5 seconds. With the forearm in a pronated position, monofilaments were placed on the skin between the styloid process of the radius and the ulnar in the forearm (Figure 1(b)). Patients were required to close their eyes. Placement began through application of the smaller filaments to the skin; placement continued to the larger filaments when patients did not respond. The point at which patients perceived the contact of their forearm with the filament was measured. Measurements were first obtained from the nonparetic side.

\subsection{Statistical Analysis}

We analyzed the data using the Mann-Whitney $U$ test to differentiate between the nonparetic and paretic side thresholds by gender. The differences in the nonparetic and paretic side thresholds were also analyzed with the Wilcoxon test in paired comparisons. Correlations between the electrical sensory and the light-touch thresholds were determined using Pearson's test correlation coefficient. The data are expressed as means \pm standard errors (SE). A P value of $<0.05$ was considered statistically significant. SPSS Version 18.0 (International Business Machines, Armonk, USA) for Microsoft Windows was used for analysis in this study. The protocol for the study was approved by the Committee of Ethics in Research of the University of Yongin, in accordance with the terms of Resolution 5-1-20, December 2006.

\section{RESULTS}

Upon analysis of the electrical sensory threshold without distinguishing between the sexes, a significant difference existed between the nonparetic side (34.8 \pm 4.8$)$ and paretic side $(50.9 \pm 2.2)$. The electrical sensory threshold for the paretic side was also higher than for the nonparetic side $(\mathrm{P}=0.000)$ (Figure 2(a1)). A significant difference materialized between male $(37.5 \pm 1.7)$ and female $(32.1 \pm 0.9)$ when the electrical sensory threshold was measured for the nonparetic side; this threshold was significantly higher in male than in female $(\mathrm{P}=0.021)$ (Figure 2(a2)). A significant difference between male and female for this test also surfaced on the paretic side (male, $55.7 \pm 2.9$; female, $46.1 \pm 2.9 ; \mathrm{P}=0.016$ ) (Figure 2(a2)). Meanwhile, when the electrical pain threshold was analyzed without taking sex into account, a significant difference existed between the nonparetic side (73.2 $\pm 2.4)$ and paretic side $(86.6 \pm 1.9)$. The electrical pain threshold for the paretic side was higher than for the nonparetic side $(\mathrm{P}=0.000)$ (Figure 2(b1)). When the electrical pain threshold was analyzed by sex, no signifycant difference existed between male $(75.4 \pm 1.0)$ and female $(71.1 \pm 3.5)$ on the nonparetic side. However, the electrical pain threshold of male was higher than that for female on the nonparetic side $(\mathrm{P}=0.051)$ (Figure 2(b2)). When the electrical pain threshold was analyzed by sex, no significant differences existed between the male (90.0 $\pm 2.2)$ and female $(83.3 \pm 2.9)$ on the paretic side. However, the electrical pain threshold of male was higher than female on the paretic side $(P=0.10)$ (Figure 2(b2)). In an analysis of the light-touch threshold without differentiating for sex, a significant difference existed between the nonparetic side $(2.9 \pm 0.0)$ and paretic side (4.3 $\pm 0.4)$; the light-touch threshold for the paretic side was higher than that for the nonparetic side $(\mathrm{P}=0.002)$ (Figure 2(c1)). When sex was taken into account for the light-touch threshold, no significant difference existed between the male $(2.8 \pm 0.0)$ and female $(2.9 \pm 0.1)$ on the nonparetic side, but the light-touch threshold of female was higher than that in male for the nonparetic side $(\mathrm{P}=0.695)$ (Figure 2(c2)). There was no significant difference between the male $(4.0 \pm 0.4)$ and female $(4.7 \pm$ $0.6)$ on the paretic side; however, the light-touch threshold of female was higher than male in paretic side ( $\mathrm{P}=$ 0.556) (Figure 2(c2)). The electrical sensory and pain threshold were significantly correlated with the lighttouch threshold in paretic compared with those nonparetic side, respectively (Figures 2(d1) and (d2)).

\section{DISCUSSION}

The present study demonstrates for the first time that differences of sensory threshold exist between the paretic and nonparetic sides of hemiplegic patients, including the clinically important sensory assessment. Previous studies have reported the sensory threshold in healthy volunteers [19,21,22]. In our present study, the overall thresholds for sensory impairment were higher on the nonparetic side compared to the paretic side for hemiplegic patients. Differences according to sex also surfaced. Men demonstrated a significantly higher electrical sensory threshold than did women in both the nonparetic and paretic sides. The results of our present study agree with the findings of some authors who have found that men have a higher sensory threshold than woman [21,22]. Likewise, the electrical pain thresholds for both the nonparetic and paretic sides were higher for men than for women, another result that replicates results reported by previous researchers $[27,28]$. Even if pain is not the major focus of this study, these findings would be of interest to those who wish to further evaluate the pain threshold of electrical stimulation. Researchers have assumed that the higher sensory pain thresholds of men are related to 


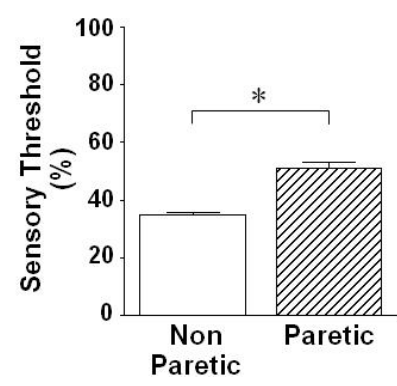

(a1)

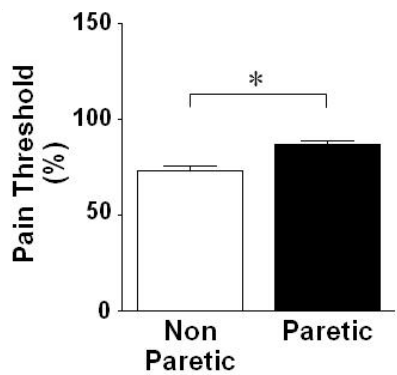

(b1)

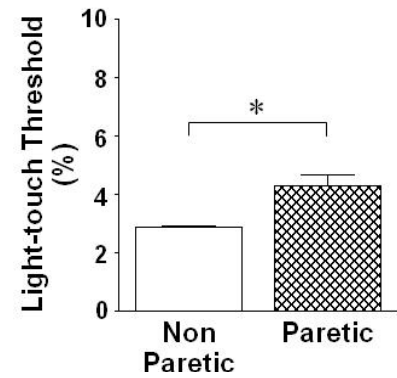

(c1)

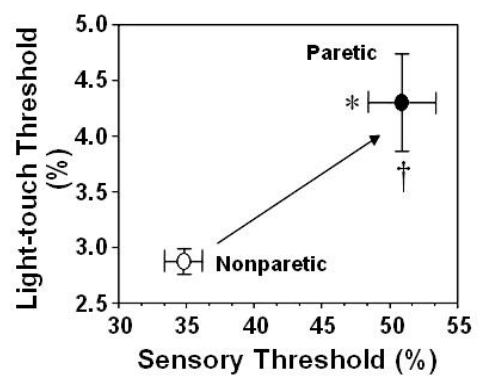

(d1)

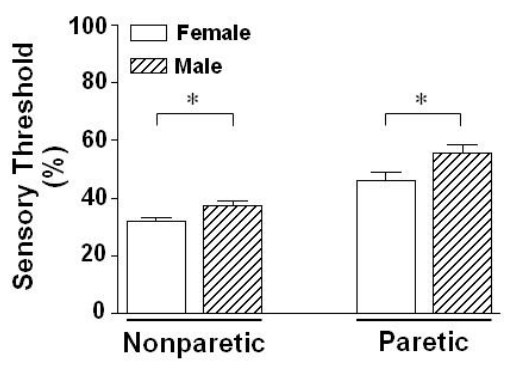

(a2)

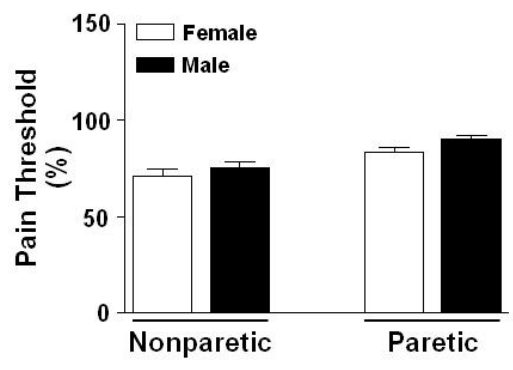

(b2)

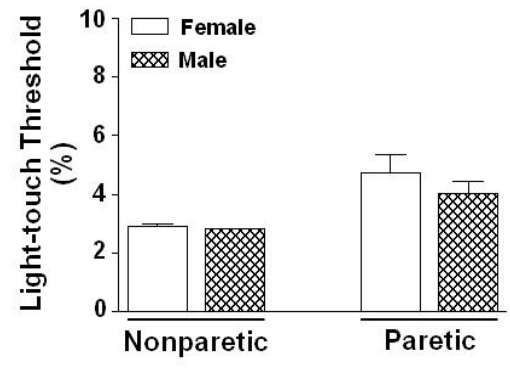

(c2)

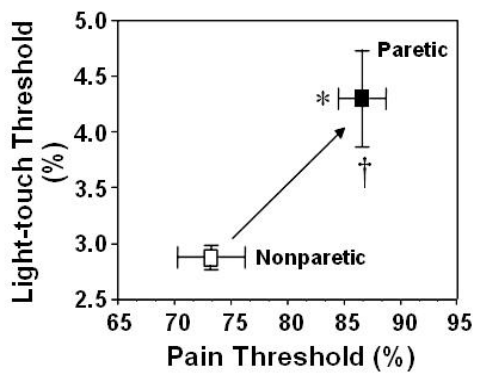

(d2)

Figure 2. Difference in the stimulation-induced sensory thresholds between paretic and nonparetic sides of patients with after stroke.

the variety or type of stimulation [29], hair distribution and shaving [30], epidermal nerve fiber density [31], skin temperature, and skinfold thickness [22]. Previous studies using other types of stimulation methods suggested differences in sensory thresholds between genders associated with differences in epidermal nerve fiber density and skinfold thickness [22,32,33]. Cadaver and skin biopsy studies have reported that women have higher epidermal nerve fiber density than men [34,35]. In women, therefore, the greater sensitivity to transcutane- ous electrical stimulation could be analyzed by gender differences in the morphology and/or density of epidermal nerve fibers, although gender-related differences in hormone concentrations (progesterone in particular) cannot be excluded $[34,36]$. Interestingly, the lower thresholds reported were correlated with greater subcutaneous fat tissue thickness in participants (more likely to be women), therefore indicating that the individual sensory response can be forecast from subcutaneous fat tissue thickness [22]. Previous studies have had results in 
accordance with our findings that the light-touch threshold for the paretic side was higher than for the non-paretic side, and there was no significant difference according to sex [21]. This general association between lesion size on imaging and tactile impairment has been discovered in studies of other cerebral defects $[37,38]$. Additionally, the density of sensory receptors can vary between individual studies, and between different ages of individuals in specific studies [39,40]. Meissner's corpuscles are located on various areas of the skin [41]. It is sensitive to light touch [42]. Meissner's corpuscles also exhibit structural modifications and general decline in amount and cross-sectional area with aging [43]. According to our results, no correlation existed between the electrical sensory threshold and the light-touch threshold. This can be explained by the fact that the sensory quality of light-touch is most related to superficial touch, while electric sensory testing is related more to subcutaneous touch. There are some limitations to this study. Data are not provided related to differences between a patient group and healthy groups for sensory and pain thresholds. However, a previous study of 30 hemiplegic patients reported a higher light-touch threshold for nonparetic hands in patients than was reported in the hands of normal subjects [20]. This study is significant because, for the first time, these tests were applied to both the paretic and nonparetic sides of hemiplegic patients. The results obtained in this study extend our previous findings and further suggest that not only electrical stimulation but also other stimulation is needed for further study of and assessment in patients after stroke. In conclusion, this study will help in the evaluation of sensory and pain thresholds for rehabilitation in hemiplegic patients.

\section{REFERENCES}

[1] Anderson, E.K. (1971) Sensory impairments in hemiplegia. Archives of Physical Medicine and Rehabilitation, 52, 293-297.

[2] Borstad, A., Schmalbrock, P., Choi, S. and Nichols-Larsen, D.S. (2012) Neural correlates supporting sensory discrimination after left hemisphere stroke. Brain Research, 1460, 78-87. doi:10.1016/j.brainres.2012.03.060

[3] Nelles, M., et al. (2008) Diffusion tensor pyramidal tractography in patients with anterior choroidal artery infarcts. American Journal of Neuroradiology, 29, 488-493. doi:10.3174/ajnr.A0855

[4] Schaechter, J.D., et al. (2006) Structural and functional plasticity in the somatosensory cortex of chronic stroke patients. Brain, 129, 2722-2733. doi:10.1093/brain/awl214

[5] Wakana, S., et al. (2004) Fiber tract-based atlas of human white matter anatomy. Radiology, 230, 77-87. doi:10.1148/radiol.2301021640

[6] Rose, L., et al. (1994) Tactile extinction and functional status after stroke: A preliminary investigation. Stroke, 25, 1973-1976. doi:10.1161/01.STR.25.10.1973

[7] Kim, M.Y., et al. (2012) The effects of functional electrical stimulation on balance of stroke patients in the standing posture. Journal of Physical Therapy Science, 24, 7781. doi:10.1589/jpts.24.77

[8] Kusoffsky, A., Wadell, I. and Nilsson, N.Y. (1982) The relationship between sensory impairment and motor recovery in patients with hemiplegia. Scandinavian Journal of Rehabilitation Medicine, 14, 27-32.

[9] Aqlioti, S., et al. (1996) Thumb-pointing in humans after damage to somatic sensory cortex. Experimental Brain Research, 109, 92-100.

[10] Yekutiel, M. and Guttman, E. (1993) A controlled trial of the retraining of the sensory function of the hand in stroke patients. Journal of Neurology, Neurosurgery \& Psychiatry, 56, 241-244. doi:10.1136/jnnp.56.3.241

[11] Sommerfeld, D.K. and von Arbin, M.H. (2004) The impact of somatosensory function on activity performance and length of hospital stay in geriatric patients with stroke. Clinical Rehabilitation, 18, 149-155. doi:10.1191/0269215504cr710oa

[12] Tyson, S.F., et al. (2008) Sensory loss in hospital admitted people with stroke: Characteristics, associated factors, and relationship with function. Neurorehabilitation and Neural Repair, 22, 166-172. doi:10.1177/1545968307305523

[13] Leeanne, M. and Thomas, A. (2001) Frequency of discriminative sensory loss in the hand after stroke in a rehabilitation setting. Journal of Rehabilitation Medicine, 43, 257-263.

[14] Patel, A.T., et al. (2000) The relation between impairments and functional outcomes poststroke. Archives of Physical Medicine and Rehabilitation, 81, 1357-1363. doi:10.1053/apmr.2000.9397

[15] Kim, J.S. and Choi-Kwon, S. (1996) Discriminative sensory dysfunction after unilateral stroke. Stroke, 27, 677682. doi:10.1161/01.STR.27.4.677

[16] Gracely, R.H., et al. (1988) A multiple random staircase method of psychophysical pain assessment. Pain, 32, 5563. doi:10.1016/0304-3959(88)90023-1

[17] Sang, C.H., Mitchell, B.M. and Gracely, R.H. (2003) Stability and reliability of detection thresholds for human A-beta and A-delta sensory afferents determined by cutaneous electrical stimulation. Journal of Pain and Symptom Management, 25, 64-73. doi:10.1016/S0885-3924(02)00541-9

[18] Phyllis, M. (1997) Sensory function assessment: A pilot comparison study of touch pressure threshold with texture and tactile discrimination. Journal of Hand Therapy, 10, 24-28.

[19] Lund, I., et al. (2005) Evaluation of variations in sensory and pain threshold assessments by electrocutaneous stimulation. Physiotherapy Theory and Practice, 21, 81-92. doi:10.1080/09593980590922307

[20] Jeon, H.J., Kim, J.H., Kim, B. and Kim, J. (2012) Analysis of high-frequency transcutaneous electrical nerve stimulation-induced sensory threshold from the elderly peo- 
ple for healthy life. Toxicology and Environmental Health Sciences, 4, 167-172. doi:10.1007/s13530-012-0132-7

[21] Leong, G.W., Lauschke, J., Rutowski, S.B. and Waite, P.M. (2010) Age, gender, and side differences of cutaneous electrical perceptual threshold testing in an ablebodied population. Journal of Spinal Cord Medicine, 33, 249-255.

[22] Maffiuletti, N.A., et al. (2011) Effect of gender and obesity on electrical current thresholds. Muscle Nerve, 44, 202-207. doi:10.1002/mus.22050

[23] Weinstein, S., Semmes, J., Ghent, L. and Teuber, H.L. (1958) Roughness discrimination after penetrating brain injury in man: Analysis according to locus of lesion. Journal of Comparative \& Physiological Psychology, 51, 269-275. doi:10.1037/h0047187

[24] Essing, J.P., Gersten, J.W. and Yarnell, P. (1980) Light touch thresholds in normal persons and cerebral vascular disease patient: Bilateral deficit after unilateral lesion. Stroke, 11, 528-533. doi:10.1161/01.STR.11.5.528

[25] Corkin, S., Milner, B. and Rasmussen, T. (1970) Somatosensory thresholds: Contrasting effects of postcentral gyrus and posterior parietallobe excisions. Archives of Neurology, 23, 41-58. doi:10.1001/archneur.1970.00480250045007

[26] Carey, L.M. (1995) Somatosensory loss after stroke. Critical Reviews in Physical and Rehabilitation Medicine, 7, 51-91.

[27] Fillingim, R.B. (2000) Sex, gender, and pain: Women and men really are different. Current Review of Pain, 4, 24-30. doi:10.1007/s11916-000-0006-6

[28] Riley, J.L. 3rd, et al. (1998) Sex differences in the perception of noxious experimental stimuli: A meta-analysis. Pain, 74, 181-187. doi:10.1016/S0304-3959(97)00199-1

[29] Lautenbacher, S. and Rollman, G.B. (1993) Sex differences in responsiveness to painful and non-painful stimuli are dependent upon the stimulation method. Pain, 53, 255-264. doi:10.1016/0304-3959(93)90221-A

[30] Caissie, R., et al. (2007) Quantitative method to evaluate the functionality of the trigeminal nerve. Journal of Oral and Maxillofacial Surgery, 65, 2254-2259. doi:10.1016/j.joms.2006.09.010

[31] Gøransson, L.G., Mellgren, S.I., Lindal, S. and Omdal, R. (2004) The effect of age and gender on epidermal nerve fiber density. Neurology, 62, 774-777. doi:10.1212/01.WNL.0000113732.41127.8F
[32] Leitgeb, N., Schroettner, J. and Cech, R. (2005) Electric current perception of the general population including children and the elderly. Journal of Medical Engineering \& Technology, 29, 215-218. doi:10.1080/03091900412331291705

[33] Irnich, W. and Batz, L. (1989) The perception threshold for $50 \mathrm{~Hz}$ alternating voltage and current. Biomedical Technician (Berlin), 34, 207-209. doi:10.1515/bmte.1989.34.9.207

[34] Bakkers, M., et al. (2009) Intraepidermal nerve fiber density and its application in sarcoidosis. Neurology, 73, 1142-1148. doi:10.1212/WNL.0b013e3181bacf05

[35] Mowlavi, A., et al. (2005) Increased cutaneous nerve fibers in female specimens. Plastic and Reconstructive Surgery, 116, 1407-1410. doi:10.1097/01.prs.0000182339.83156.06

[36] Roglio, I., et al. (2008) Neuroactive steroids and peripheral neuropathy. Brain Research Reviews, 57, 460-469. doi:10.1016/j.brainresrev.2007.04.010

[37] Campbell, J.K., et al. (1978) Computed tomography and radionuclide imaging in the evaluation of ischemic stroke. Radiology, 126, 695-702.

[38] Yarnell, P., Monroe, P. and Sobel, L. (1976) Aphasia outcome in stroke: A clinical neuroradiological correlation. Stroke, 7, 516-522. doi:10.1161/01.STR.7.5.516

[39] Baumann, K.I., Hamann, W. and Leung, M.S. (1986) Mechanical properties of skin and responsiveness of slowly adapting type I mechanoreceptors in rats at different ages. The Journal of Physiology, 371, 329-337.

[40] Ras, V.R. and Nava, P.B. (1986) Age-related changes of neurites in Meissner corpuscles of diabetic mice. Experimental Neurology, 91, 488-501. doi:10.1016/0014-4886(86)90047-6

[41] Cauna, N. and Ross, L.L. (1960) The fine structure of Meissner's touch corpuscles of human fingers. Journal of Biophysical and Biochemical Cytology, 8, 467-482. doi:10.1083/jcb.8.2.467

[42] Bolton, C.F., Winkelmann, R.K. and Dyck, P.J. (1964) A quantitative study of Meissner's corpuscles in man. Transactions of the American Neurological Association, 89, 190-192.

[43] Bruce, M.F. (1980) The relation of tactile thresholds to histology in the fingers of elderly people. Journal of Neurology, Neurosurgery \& Psychiatry, 43, 730-734. doi:10.1136/jnnp.43.8.730 